\title{
New directions for artificial cells using rapid prototyped biosystems
}

\author{
Mark S. Friddin, ${ }^{* a}$ Yuval Elani, ${ }^{\text {abc }}$ Tatiana Trantidou ${ }^{\mathrm{a}}$ and Oscar Ces*abc \\ aDepartment of Chemistry, Imperial College London, Wood Lane, London, W12 0BZ, UK. \\ 'Institute of Chemical Biology, Imperial College London, Wood Lane, London, W12 0BZ, UK. \\ ${ }^{\mathrm{c}}$ FABRICELL, Molecular Sciences Research Hub, Imperial College London, Wood Lane, London, W12 0BZ, UK.
}

\begin{abstract}
Microfluidics has been shown to be capable of generating a range of single- and multi- compartment vesicles and bilayer delineated droplets that can be assembled in 2D and 3D. These model systems are becoming increasingly recognized as powerful biomimetic constructs for assembling tissue models, engineering therapeutic delivery systems and for screening drugs. One bottleneck in developing this technology is the time, expertise and equipment required for device fabrication. This has led to interest across the microfluidics community in using rapid prototyping to engineer microfluidic devices from Computer Aided Design (CAD) drawings. We highlight how this rapid prototyping revolution is transforming the fabrication of microfluidic devices for bottom-up synthetic biology. We provide an outline of the current landscape and present how advances in the field may give rise to the next generation of multifunctional biodevices, particularly with Industry 4.0 on the horizon. Successfully developing this technology and making it open-source could pave the way for a new generation of citizen-led science, fueling the possibility that the next multibillion dollar start-up could emerge from an attic or a basement.
\end{abstract}

Microfluidics has become established as a versatile platform technology for miniaturizing and integrating fluid handling and sample preparation inside microchannels engineered within credit card-sized chips. This powerful ability combined with the option of high-throughput has driven the uptake of microfluidics into a range of applications that span the life sciences. ${ }^{1,2}$ This includes the development of devices for on-chip cell culture, ${ }^{3}$ flow cytometry, ${ }^{4}$ organ on-chip, ${ }^{5}$ integrated biosensing and point-of-care diagnostics. ${ }^{6}$ Another field that has been totally transformed by the introduction of microfluidics is synthetic biology, ${ }^{7,8}$ a discipline that is dedicated to imparting biological function either by modifying biological existing cells (topdown synthetic biology) or by engineering artificial cells/protocells from non-living building blocks (bottom-up synthetic biology). To this end, microfluidics has enabled the construction of devices dedicated to the directed evolution of proteins, ${ }^{9}$ the $3 \mathrm{D}$ culture of mesenchymal stem cells, ${ }^{10}$ and the fabrication of artificial cells of defined architectures. ${ }^{11}$ Here, microfluidics has had a particularly transformative impact as it has enabled constructs to be assembled that cannot be reasonably engineered in any other way. For example, multicompartment artificial cells are difficult to construct manually off-chip at biomimetic length-scales and in a controlled manner, while the high throughput offered by microfluidics can be exploited to connect thousands of independent subunits into highly packed minimal tissue systems. ${ }^{12}$

However, one key problem is that building these devices traditionally requires expertise in microfabrication, access to a dedicated cleanroom facility equipped for soft lithography, and a number of expensive consumables including photomasks, photoresists and either silicon or glass wafers. Critically, fine spatial control of channel wettability is also required when engineering dual-phase systems for the construction of emulsified multicompartment architectures and a toolkit consisting of at least tubing, interconnects and syringe pumps are required to operate the final device. These factors, coupled with the relatively slow turnaround in device iterations, severely limit the full-scale adoption of this technology in synthetic biology and other fields alike.

This bottleneck in fabrication has motivated the uptake of several different rapid prototyping (RP) technologies for the production of microfluidic chips in recent years, each offering the ability to construct devices from Computer Aided Designs (CAD) inside a few hours, without the need for expensive materials or dedicated laboratories. One example that has revolutionized the aeronautic ${ }^{13}$ and automotive ${ }^{14}$ industries and become a key player in a host of medical applications ${ }^{15}$ including prosthetics and orthotics, ${ }^{16}$ dentistry, ${ }^{17}$ and the production of surgical models, ${ }^{18}$ is $3 \mathrm{D}$ printing (also known as additive manufacturing). Computer numerical control (CNC) milling and flatbed laser cutting are other approaches that are starting to gain traction in the RP of setups and devices, ${ }^{19}$ while there has also been a renaissance in the use of lamination to assemble chips from layers of plastic or dry film resist. ${ }^{20}$

These approaches all offer the potential of cleanroom-free microfluidics to the lab bench in hours without the need for specialist infrastructure. This speed coupled with (at least) part-automation of the process also means that, in principle, devices with greater complexity can be engineered as there is a quicker turnaround between part generations due to less time being spent in the cleanroom. The ever-increasing affordability of these pieces of equipment together with the wide availability of materials and open-source repositories for designs and materi$\mathrm{als}^{21}$ is also starting to forge a new 


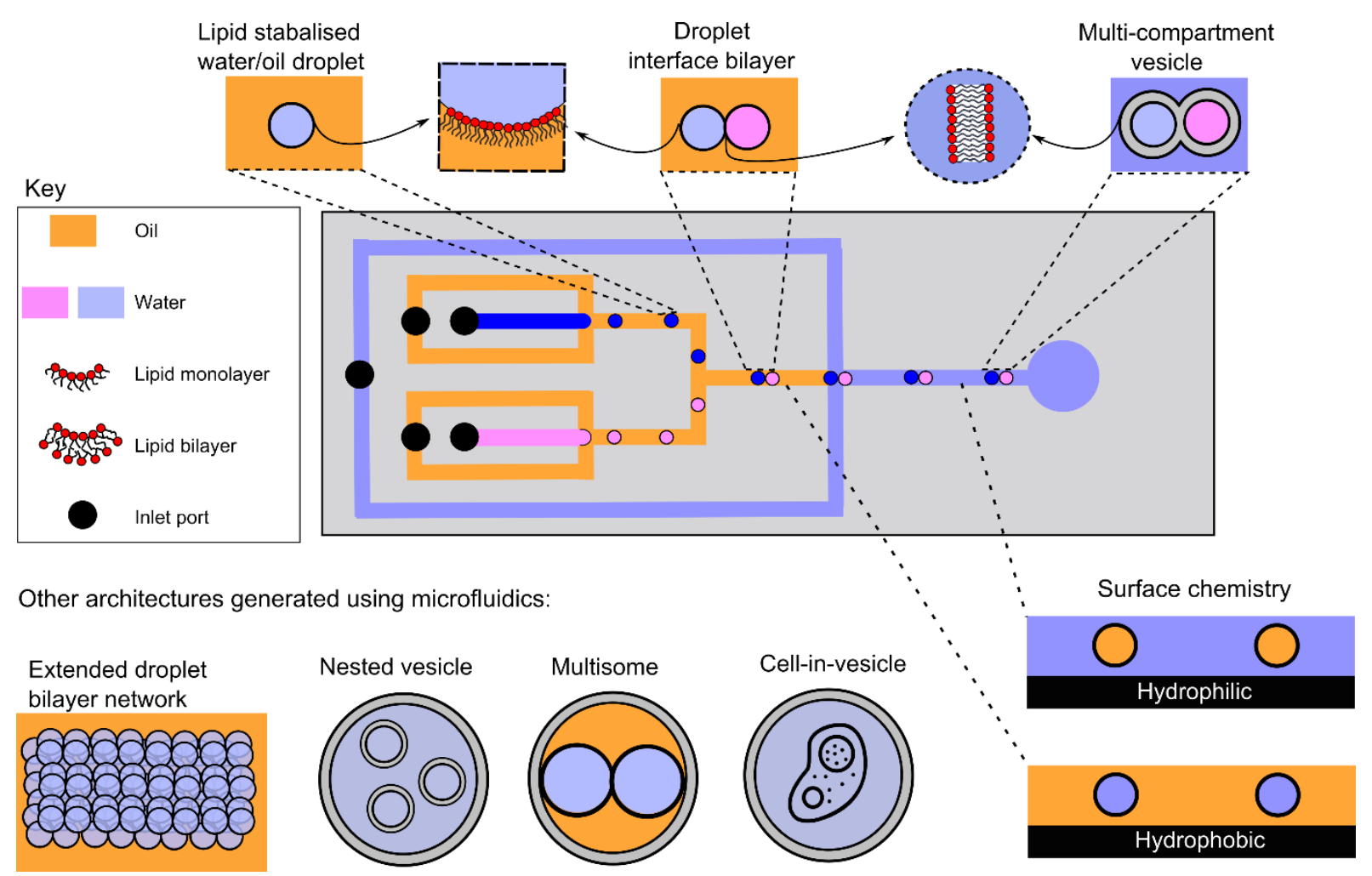

Figure 1. The microfluidic generation of different model architectures on-chip. A lipid-monolayer stabilized water-in-oil droplet is typically generated at either a flow focusing or T-junction. Water droplets from two different inlets can be focused into contact to form a droplet interface bilayer (DIB). These are monolayer coated droplets, which form a bilayer where two droplets meet. DIBs can be passed through a phase-transfer junction, from an oil to an aqueous phase, to form multi-compartment vesicles with a fully encasing bilayer. The external oil phase can be removed either by a hydrophobic waste channel (not shown), or through dissolution into the aqueous phase and subsequent evaporation, depending on the oils solubility properties. Different configurations of the microfluidics can enable the formation of extended DIB networks, nested vesicles, multisomes and cells-in-vesicles. Channel dimensions are generally ca. $100 \mu \mathrm{m}$ wide and generate droplets ca. $100 \mu \mathrm{m}$ in diameter. The microfluidic device is typically the size of a standard $75 \mathrm{x} 25 \mathrm{~mm}$ glass microscope slide. Channel surface chemistry should be either hydrophilic or hydrophobic if the continuous (i.e. external) phase is water or oil respectively. Droplets are encased by a lipid monolayer or bilayer as indicated. Scheme is not to scale.

class of citizen-led science, ${ }^{22}$ fueling the possibility that startup SynBio companies could emerge from a home living room, garage or basement. In place and ready to support such enterprise are an increasing number of public biohackspaces (Genspace, DIY Bio, London Biohackspace, GaudiLabs/OpenDrop, Open Cell) in addition to Incubators and non-university led spin-off initiatives.

With next generation 3D printers set to offer the field of medicine a new class of heart valves, ${ }^{23}$ cartilage, ${ }^{24}$ bone $^{25}$ and implantable biosensors with integrated electronics, ${ }^{26}$ we question how RP techniques can have a similar paradigm-shifting impact in the field of bottom-up synthetic biology. This article explores this emerging field of bio-manufacturing and outlines the challenges that need to be overcome in order for the next generation of devices to be entirely rapid prototyped or rapid manufactured. Realizing this potential could democratize microfluidics for synthetic biology, paving the way for the next generation of artificial tissue constructs, smart biosensors and compartmentalized bioreactors which could be manufactured remotely and automatically on-demand.

\section{BOTTOM-UP SYNTHETIC BIOLOGY: STATE-OF- THE-ART}

In recent years, bottom-up synthetic biology, and specifically the construction of membrane-bound artificial cells and celllike devices, has exploded from patch clamped black lipid membranes (BLMs) and liposomes, to communicating multicompartment artificial cells ${ }^{27}$ and extended bilayer networks, consisting of thousands of interconnected lipid bilayers. ${ }^{28} \mathrm{At}$ the core of these achievements is the generation of lipid stabilized water-in-oil microdroplets. These lipid-coated droplets in oil can be placed in contact to form droplet interface bilayers (DIBs) $)^{29,30}$ or transferred into an aqueous medium to form vesicles. ${ }^{31}$ Vesicles, also known as liposomes, are lipid bilayer encased fluid containers that are suspended in an aqueous environment. Vesicles can be compartmentalized by engulfing multiple DIB connected water-in-oil droplets in a bilayer either offchip ${ }^{32}$ or, on-chip ${ }^{33}$ or by embedding smaller vesicles inside to form nested structures. ${ }^{34,35}$ When compartments are stabilized by an intermediate oil phase, with a bilayer present at all waterwater interfaces, they are known as multisomes. ${ }^{36}$ These can also be formed using microfluidic methods. . ${ }^{37-39}$ These structures are depicted in Fig. 1.

Compartmentalized vesicles or their analogs have been engineered with pores to facilitate the controlled exchange of components between compartments ${ }^{27}$ and assembled from lightsensitive lipids that can be externally triggered to drive enzymatic reactions. ${ }^{34}$ Vesicles can also be controllably fused together to deliver material payloads, ${ }^{40}$ locally heated to exhibit phase-transitions, ${ }^{31}$ coupled with pores and blockers for programmable leakage ${ }^{41}$ and have growing applications as smart drug delivery systems. ${ }^{42}$ There have also been recent advances 
in the assembly and functionalization of DIB networks, including the 3D bioprinting of 'tissue-like materials' from thousands of DIBs assembled in $3 \mathrm{D},{ }^{28}$ and the ability to trigger protein expression in selected droplets using light. ${ }^{43,44}$ Microfluidics also allows encapsulation of cells in vesicles, ${ }^{45,46}$ which can also be considered a form of compartmentalization. This paves the way for the construction of 'hybrid' artificial cells, where the encapsulated 'living' compartment acts an organelle within a vesicle-based synthetic cell. ${ }^{46}$

These achievements have totally transformed the landscape of bottom-up synthetic biology. To stretch this technology further, membranes need to be functionalized with a wider range of proteins, composed of more bio-representative lipids and formed from droplets that can significantly vary in content over extended networks. Most critically, these techniques require seamless uptake across the life sciences, something that can only be achieved once device fabrication is sufficiently streamlined to enable end-users to focus on developing the intended application.

\section{RAPID PROTOTYPING TECHNOLOGIES FOR ENGINEERING MICROFLUIDIC DEVICES}

With hackspaces, fab labs and maker spaces opening up all over the world, interdisciplinary researchers have never had greater access to RP technologies for building setups and parts. However, engineering microfluidic chips is a specialist task that demands the generation of fluidically sealed or easily bondable devices at high resolution. There is also a need to control the wettability of the channel surface when constructing multiphase systems. Naturally, all RP approaches for synthetic biology applications are compared to soft lithography, where elastomeric devices are typically cast from an SU-8 master and bonded to a glass microscope slide. The smallest channel width achievable is approximately $10 \mu \mathrm{m}$ (with much smaller depths attainable but not reproducibly), however multi-layered devices require further processing, additional photomasks and alignment. This is in addition to the beforementioned infrastructure, expertise and consumables. These factors lead to a trade-off between fabrication approaches, usually in the form of resolution and cost versus speed. While there are a host of different RP technologies available, the approaches for engineering microfluidic devices can be broadly divided as follows (Fig. 2);

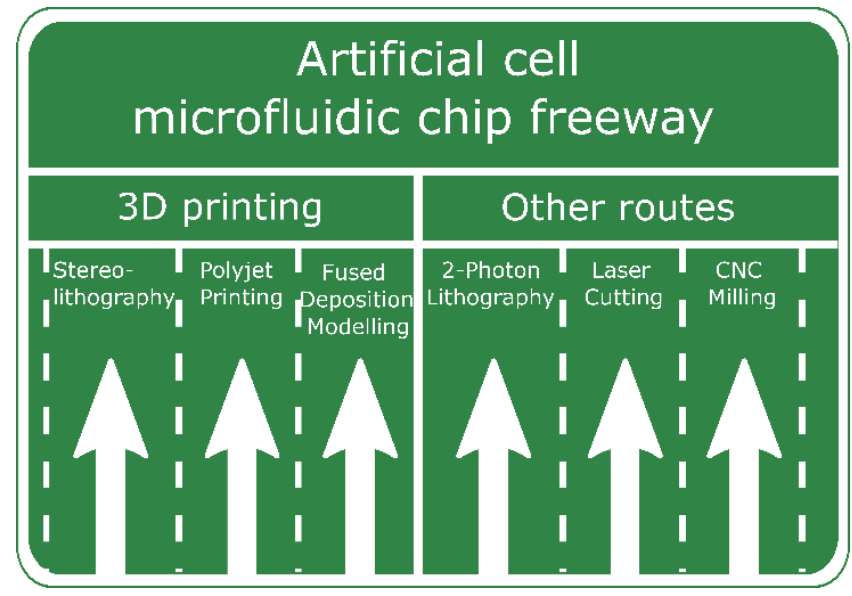

Figure 2. Different routes for rapid prototyping microfluidic devices for bottom-up synthetic biology

Computer Numerical Controlled (CNC) milling: A subtractive method that uses an end mill under computerized numerical control to remove material. Usually performed with plastics such as cyclic olefin copolymer (COC), Polycarbonate (PC) or poly(methyl methacrylate) (PMMA) to make microfluidic devices directly, ${ }^{47,48}$ or as masters for casting devices from elastomers. ${ }^{49}$ The method typically relies on the generation of a Computer Aided Manufacturing (CAM) file from a CAD design and requires specialist user handling. The channel dimensions are defined by the size of the end mill installed, which is typically no smaller than $0.1 \mathrm{~mm}$ in diameter. The key advantages of this approach are: no photolithography is required, the process is repeatable, channel dimensions are uniform and accessible for downstream surface treatment. The key disadvantages are: limit to resolution, end mills often break, devices must be bonded, square endmills are required to engineer square features, mills need to run at high speed (100,000 RPM) to accommodate small end mills, device fabrication is slow, requires supervision and can require heavy optimization.

3D printing: There are a host of different 3D printing technologies available for producing microfluidic chips that have been reviewed in detail elsewhere. ${ }^{50-53}$ In summary, all technologies share the fact that parts are built additively (i.e. layer by layer). This is primarily achieved in three different ways; 1) By melting and depositing thermoplastics such as ABS (acrylonitride butadiene styrene), PLA (polylactic acid) and COC on an adjustable build platform in a technique known as fused deposition modelling (FDM) 2) By inkjet printing liquid UV photocurable resins on an adjustable build platform (polyjet printing). 3) By immersing a retractable baseplate in a vat of UV photocurable resin and curing layer by layer using a laser (stereolithography, SLA), a process that can be assisted using digital light processing (DLP-SLA). There is a number of advantages and disadvantages using each approach; FDM printers are typically very economical and there is a wide range of well characterized, transparent and biocompatible materials available, however, they tend to suffer from poor print resolution (it is difficult to engineer channels smaller than $250 \mu \mathrm{m}$ ). DLP-SLA offers excellent speed and resolution $(100 \mu \mathrm{m}$ wide channels are achievable), but devices can only be made of one material (usually proprietary) and uncured resin must be fully expelled from the $3 \mathrm{D}$ printed channels. Polyjet printers offer the ability to print multiple different materials, including a water soluble support material, in a process that requires little user optimization. However, these printers are typically expensive $(\$ 10 \mathrm{k}-$ $\$ 100 \mathrm{k}+$ ), the resolution is similar to FDM and the soluble support material can be difficult to remove from enclosed channels. Overall, the key advantages shared by all 3D printing techniques include; high speed, process automation, limited lab space required and the ability to share designs. The drawbacks include high process variability, general inability to pause printing for surface modification, and the common requirement for significant user optimization. All 3D printers are also sensitive to the direction in which the object is constructed, require expertise in CAD and the use of a slicer to convert an STL file into layer by layer instructions for printing. Some systems also require parts to be post processed after printing.

Flatbed laser cutting: A widely available technique that can cut or engrave plastics (typically PMMA) from a 2D design. The process is rapid and is becoming popular in applications with sessile droplets, however, engraved surfaces appear rounded due to the parabolic profile of the laser and the microstructure of the channels is coarse. Chips built for continuous flow need to be sealed, which can be tedious. Among many bonding options is a combination of chemical solvent bonding and thermal bonding, which can be realized in-house using a 
commercial microwave. ${ }^{54}$ Double-sided pressure-sensitive tapes $^{55}$ or laminating dry film resists ${ }^{56}$ are another low-cost solutions for bonding laser-cut microfluidic devices. The key advantages of this method are speed and availability coupled with the fact that parts can be made from simple 2D designs meaning that $3 \mathrm{D}$ CAD is not required. The drawbacks include the requirement to seal devices, the parabolic shape of the laser and the limitation of the number of materials that can be used.

2-photon lithography: 2-photon lithography or direct laser writing enables the fabrication of three-dimensional micro- and nanostructures using photo-sensitive polymers and a non-linear two-photon absorption process. ${ }^{57}$ An ultra-short pulse laser is focused into the resin and triggers a two-photon polymerization only in the focal of volume (the 3D analogon of a pixel). 2photon polymerization allows highly accurate, reproducible, maskless 3D designs from the nano- $(>150 \mathrm{~nm})$ to the micro- $(1$ $-1000 \mu \mathrm{m})$ and the meso-scale $(<2000 \mu \mathrm{m})$. Over the last decade this technology has been commercialized and has set a new benchmark for microfabrication and additive manufacturing, with particular impact on the field of microfluidics. The technology has enabled the rapid prototyping of different microfluidic elements including filters, mixers, capillary pumps, microfluidic traps, ${ }^{58}$ microconnectors and customized nozzles, ${ }^{59}$ overcoming the technical challenges of custom 3D printing technologies such as fabrication of nanosized feature sizes $(<1$ $\mu \mathrm{m})$ and high-aspect ratio patterns. The technology can also be employed to create hybrid devices, combining conventional microfluidic fabrication methods for large features and 2-photon lithography for smaller features. ${ }^{60}$ Commercially available systems are user-friendly and have significantly increased the speed and precision of writing compared to previous versions, however, the remaining drawbacks of this technology include the high printing time when fabricating structures in the mesoand macroscale.

\section{RP DEVICES FOR MANUFACTURING LIPID BILAYERS}

Despite the availability of RP solutions, the use of these methods to fabricate entire devices for the purpose of assembling artificial cell chassis, such as vesicles and DIB networks remains fairly limited. More commonly, these technologies have been used to create molds for chips or parts for setups. For example, King and co-workers demonstrated the use of a 3D printed master (Fig. 3A) to engineer millifluidic chips for forming bilayer delineated droplets under continuous flow ${ }^{61}$ and Sarles et al. reported the assembly of a polydimethylsiloxane (PDMS) device for forming and regulating the attachment of droplet bilayers that was made from a CNC machined mold. ${ }^{49,62}$ The same group also reported that the mold could be substituted for a $3 \mathrm{D}$ printed master to cast hydrogels (Fig. 3B). ${ }^{63}$ Laser cut acrylic parts have been similarly instrumental as wells and apertures in a number of different platforms ${ }^{64-67}$ and have also been used for engineering entire devices for assembling ${ }^{68}$ (Fig. 3C) and performing rheological analysis of DIBs using sessile droplets. ${ }^{69}$ While CNC milling has been used to fabricate entire microfluidic chips for assembling DIBs ${ }^{47}$ and DIB networks ${ }^{70}$ (Fig. 3D), the use of $3 \mathrm{D}$ printing has been less widely demonstrated for engineering devices for bottom-up synthetic biology. The Castell group reported the use of a 3D printed housing for assembling encapsulated DIBs inside capillary tubes (Fig. 3E), ${ }^{38} \mathrm{de}$ Bruin et al. showed the assembly of optically or electrically accessible lipid bilayers on hydrogel sheets encased within clear
3D printed chips (Fig. 3F), ${ }^{71}$ while the DeVoe group have recently achieved nanoliposome formation in $3 \mathrm{D}$ printed devices. ${ }^{72}$
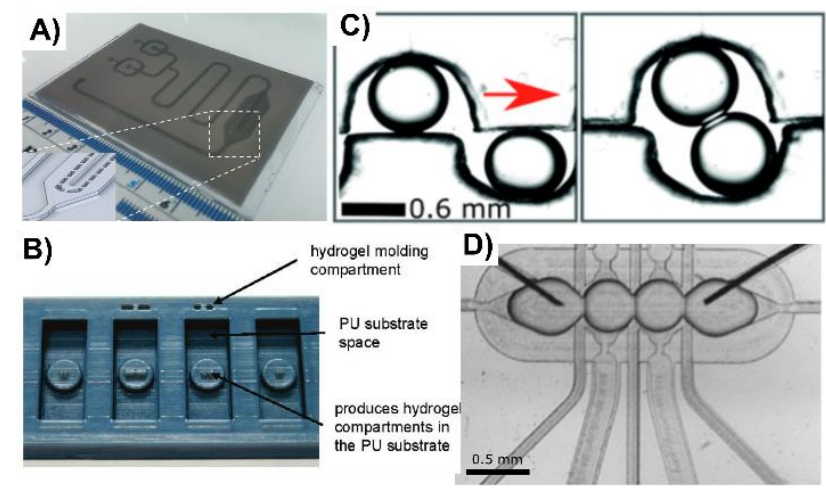

E)

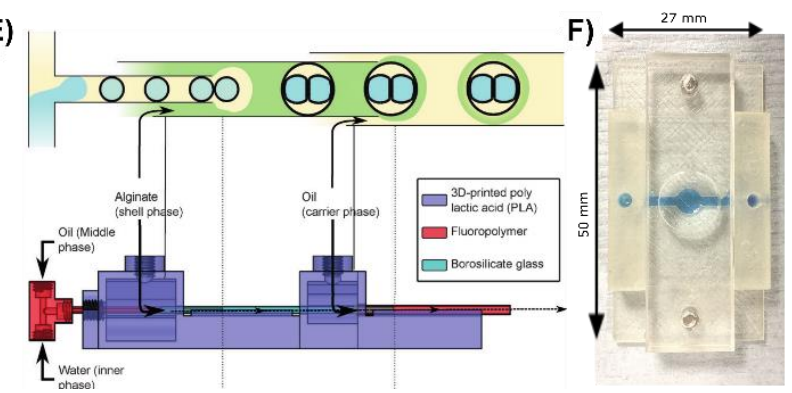

Figure 3. Different RP devices for assembling model membranes. A) A Polyjet printed master for casting a millifluidic device for assembling DIBs, reproduced from ${ }^{61}$ with permission from The Royal Society of Chemistry. B) A Polyjet printed Polyurethane (PU) mold for casting hydrogel devices for assembling DIBs. Reprinted (adapted) with permission from ${ }^{63}$. Copyright (2010) American Chemical Society. C) Laser-cut acrylic device for assembling DIBs. $\left.{ }^{68} \mathrm{D}\right) \mathrm{CNC}$ milled polycarbonate device for assembling DIBs in microfluidic channels, channel widths are 0.2 and $0.4 \mathrm{~mm}$ respectively. ${ }^{70}$ E) FDM printed assembly for the microfluidic generation of multisomes in glass capillaries. ${ }^{38}$ F) FDM printed chip for assembling droplet hydrogel bilayers. ${ }^{71}$

\section{FUTURE PERSPECTIVE}

Given recent trends it is likely that the fabrication of microfluidic devices for bottom-up synthetic biology will continue to gradually move away from conventional soft lithography and towards more open-source RP solutions. As part of this transition, it seems there will be three main phases; (i) Casting devices from RP engineered molds, (ii) Integrating RP parts in 'hybrid devices' and (iii) Exploiting RP to fabricate entire platforms. As we have highlighted, there are already some examples in the literature of phases (i) and (ii) while efforts toward phase (iii), without the use of CNC milling, tend to be limited to sessile droplets. CNC milling is highlighted here as despite its classification as an RP technology, it does not seem likely that the next generation of entirely RP engineered microfluidic platforms will be engineered in this way; the process it is not rapid, does not form sealed devices and typically requires heavy user supervision. Laser cutting is quick and automated, however it also does not produce sealed devices and well-engineered engraved channels are difficult to achieve. Unless these 
parameters can be reversed, it seems likely that the next generation of devices will be engineered using 2-photon lithography and/or additive manufacturing. Alongside the drive for optical transparency, which is already becoming a mainstream demand for devices, ${ }^{73,74}$ the critical features that will drive the development of this technology for bottom-up synthetic biology are;

Resolution: The size of bilayer encased compartments that can be engineered on-chip is directly related to the dimensions of the microchannels. This combined with growing interest in constructing multicompartment and networked model assemblies at biorepresentative length-scales, places huge emphasis on resolution when fabricating RP devices. As it stands currently no commercially available machine can compete with the unprecedented resolution offered by 2-photon lithography, however the price of these systems (ca. \$450k) and the time taken to engineer macroscopic features drive interest in quicker and more economical DLP-SLA systems which are available from as little as ca. $\$ 2 \mathrm{k}$ and can produce a chip inside 30 minutes. The drawback of this is resolution, and although there are recent reports of DLP-SLA printed channels measuring $18 \mu \mathrm{m} \times 20 \mu \mathrm{m},{ }^{75}$ this is not yet offered by commercially available machines, which are limited to ca. $100 \mu \mathrm{m}$ wide channels. It seems likely that the next generation RP solution will involve multiple techniques to craft devices, as is already starting to be realized. ${ }^{60,76}$ One powerful combination could be coupling 2-photon lithography with polyjet printing, however, for this to be feasible, incompatibilities between the two technologies need to be overcome; motorized stages for rough and fine positioning, photocurable resists with low and high resolution, adjustable optics and a universal developer. In face of the challenges, successfully realizing this could lead to a highly versatile $3 \mathrm{D}$ printing factory line that could only be improved by introducing the ability to print electronics.

Control of surface wettability: This is important when engineering dual-phase microfluidic chips for generating vesicles and multicompartment architectures. Fundamentally, surface patterning of this nature could be achieved using three different approaches; i) The surface is manually treated by the user during fabrication, ii) the device is fabricated from multiple different materials that have different surface chemistries, or iii) The device is fabricated from a new smart material that can be easily modified during fabrication, perhaps using UV light or 2-photon lithography if a suitable resist can be engineered and printed. While option i) remains viable when working with CNC milling or laser cutting, it represents a step away from the automation and fidelity offered by routes ii) and iii). There is also a compatibility issue with 3D printing as functionalizing the surface in this way would require printing to be paused, the part to be removed for treatment and returned to the printer for fabrication to resume. To our knowledge this is not an option that is currently offered by any commercially available 3D printer. Approach ii) has been shown as a viable option when fabricating chips for generating vesicles using soft-lithography, where two different types of PDMS were used. ${ }^{77}$ However for this to be incorporated into a $3 \mathrm{D}$ printing process it would require separate hydrophilic and hydrophobic materials to be deposited. It seems feasible that this could be achieved in a polyjet system. Engineering a material that offers the ability to be dynamically modified during chip fabrication is a significant challenge.

Device design and optimizing fabrication; With increasingly more complex RP fabricated chips on the horizon this will inevitably mean that greater expertise is required to produce CAD designs, especially if electronics are to be integrated. Here there is a serious risk that advancements in fabrication could be converted to complications in design, creating a similar bottleneck to what exists already. To address this concern, it is likely that more CAD packages will need to be designed especially for microfluidic applications and be coupled with artificial intelligence (AI) to assist the design process, while also instigating crosstalk with slicing software to ensure that the intended part is fabricated as per the specification. Herein there are a few major issues with print optimization and reproducibility that need to be addressed. For example, any advantages offered by the speed of fabrication are quickly circumvented if it requires the production of $10+$ chips to optimize the various printing parameters. This directly feeds into a fundamental problem in printto-print variability in parts, particularly when printing small $(<1$ $\mathrm{mm}$ ) features. Another parameter to consider for optimizing fabrication is the build environment. One issue that arises from taking-processes out of the cleanroom is the exposure to airborne particles, which will be an increasing problem as features continue to shrink. A solution to this problem could be to introduce an enclosure that provides careful control of airflow, which could be especially practical when working with small build areas. With designs set to become more complex and builds more elaborate, the open-source sharing of designs is set to play a crucial role in the development of next generation platforms.

Device integration: One of the promises of lab-on-a-chip technology is a totally integrated micro- total analysis system. While current devices continue to deliver breakthrough advances, it is fair to say that the state of the art are microsample preparation systems that require total analysis to be performed off-chip. This has created the chip-in-a-lab paradigm, largely as devices are operated in laboratories using syringe pumps under microscopes. In this context, it seems clear that the next key advancements would inclide the ability to; i) integrate micropumps and valves on-demand, and ii) fabricate instrumentation and driving electronics, both of which are areas that are continually advancing and can be compensated for currently using open-source hardware. ${ }^{78,79,80}$

Speed, affordability, and throughput: The question of how rapid is rapid? tends to evolve with time. It was only twenty years ago that soft lithography was widely considered to be an RP solution to microfluidic device fabrication, ${ }^{81}$ and it is now considered to be the benchmark for improvement. To this end, despite the abovementioned drawbacks, microfluidic devices can be printed in less than an hour using standard 3D printing methods at a cost of anywhere between \$0.1 and \$4 per chip depending on which method is used. ${ }^{50}$ It seems feasible that the status quo could change again as the field continues to develop, and as more components are integrated into device fabrication, it will be interesting to see whether the trend follows Moore's law. Furthermore, while microfluidics production of several membrane based constructs is higher-throughput that traditional one-by-one manual pipetting methods, new techniques and technologies for parallelization and scalability need to be developed to reach the throughput achieved by bulk self-assembly methods.

Automation: While many of the RP technologies discussed offer significant levels of automation compared to conventional soft lithography, it seems clear that the long-term goal is to develop an entirely automated fabrication process. Achieving this would be an unprecedented advance, bringing microfluidic device fabrication to Industry 4.0. This new paradigm (named in 
reference to the 'fourth industrial revolution') describes the use of automation, additive manufacturing / 3D printing, AI, machine learning, big data and analytics, which is expected to drive innovation and economic growth over coming decades. In the context of this article, it often relates to the production of 'smart factories' capable of automatically producing devices, and perhaps even experiments, on demand under the control of cyber physical systems. This will facilitate scale-up and reduce the reliance of skilled and experienced users further. This coupled with AI could change the face of the way that experimental science is performed, away from the lab and toward an entirely robotic factory. Such principles are already impacting the biotech industry in general and top-down synthetic biology in particular (e.g. through companies such as Transcriptic robotic cloud laboratory, and Synthace computed aided biology). When applied to RP technologies, automation could be equally transformative.

Device functionality: The next generation of RP engineered microfluidic chips for bottom-up synthetic biology should be able to manufacture multiple different bilayer-bound compartments and sub-compartments while offering the user full control of the cargo that is encapsulated. These features together with on-chip mixing and dilution should allow investigators to fully exploit microfluidics to perform complex biochemistry onboard these systems and design more bio-representative model systems and intelligent soft robotic assemblies. One key milestone toward achieving this will be to conquer the challenge of expressing different membrane proteins on-chip. This will allow membrane bound compartments to be sensitized to different inputs and allow them to sense and respond to their environments. By increasing this functionality further, it seems clear how more intelligent systems can be engineered, shifting the focus to how to assemble them with fine user 3D control. This could result in RP being used to engineer fluidic circuit boards (FCBs) that can be used as droplet printers, that used in combination with open-source hardware ${ }^{80}$ could be used to assemble large networks of multicompartment architectures with heterogeneous subunits. FCBs may comprise of multiple different layers will need to be controlled remotely via onboard electronics, which may enable them to be implanted into patients to facilitate drug delivery. Realizing a design process of this nature together with the seamless and ergonomic integration of the abovementioned advances will allow for almost any RP microfluidic platform to be generated, empowering the user to stretch the limits of their imagination when engineering new model cells, tissues and soft robotic systems.

\section{CONCLUSION}

RP technologies are having an increasingly greater impact in the bio-manufacturing of model membranes and bilayer networks but greater control over resolution and surface patterning together with greater strides toward automation is required to truly realize the potential of this platform technology. As it stands, the scene is evenly poised with soft lithography offering a significant, but safe investment of time for device fabrication, whereas the RP route can offer high-speed and economy, although the process can require heavy-user optimization. While both routes present tradeoffs and compromise, it seems clear that cleanroom-free access to microfluidics is key for the widescale adoption of this technology. The advantages are numerous if these techniques can be leveraged to fabricate chips for manufacturing multicompartment artificial cells with heterogeneous subunits, for generating cell-sized bilayer delineated droplets or for engineering other bottom-up synthetic constructs such as artificial cytoskeletons or chromosomes; paving the way for the next generation of soft robotics and artificial tissue models, while lead to new fundamental insights in cell biology. ${ }^{82}$ Integrating RP technologies with membrane-based bottom-up synthetic biology would also allow the concept of design-build-test cycle that is key to many engineering disciples to be applied to this field as well. Most critically, fully democratizing fabrication will open synthetic biology to a new wave of citizen-led science that could result in the next paradigmshifting advancement coming from an amateur scientist.

\section{AUTHOR INFORMATION}

\section{Corresponding Author}

* Email: m.friddin@imperial.ac.uk

* Email: o.ces@imperial.ac.uk

\section{ORCID}

Mark S. Friddin: 0000-0003-4421-8792

Tatiana Trantidou: 0000-0001-6784-2665

Oscar Ces: 0000-0002-6418-5644

\section{Notes}

The authors declare no competing financial interest.

\section{ACKNOWLEDGMENT}

This work was supported by the EPSRC via grants EP/J017566/1 and $\mathrm{EP} / \mathrm{K} 038648 / 1$.

\section{REFERENCES}

(1) Huebner, A.; Sharma, S.; Srisa-Art, M.; Hollfelder, F.; Edel, J. B.; deMello, A. J. Lab Chip 2008, 8, 1244-1254.

(2) David J. Beebe; Glennys A. Mensing, a.; Walker, G. M. Аnnи. Rev. Biomed. Eng. 2002, 4, 261-286.

(3) El-Ali, J.; Sorger, P. K.; Jensen, K. F. Nature 2006, 442, 403.

(4) Spencer, D.; Caselli, F.; Bisegna, P.; Morgan, H. Lab Chip 2016, $16,2467-2473$

(5) Esch, E. W.; Bahinski, A.; Huh, D. Nat. Rev. Drug Discovery 2015, 14, 248.

(6) Rackus, D. G.; Shamsi, M. H.; Wheeler, A. R. Chemical Society Reviews 2015, 44, 5320-5340.

(7) Elani, Y. Biochem. Soc. Trans. 2016, 44, 723-730.

(8) Gach, P. C.; Iwai, K.; Kim, P. W.; Hillson, N. J.; Singh, A. K. Lab Chip 2017, 17, 3388-3400.

(9) Kintses, B.; Hein, C.; Mohamed, Mark F.; Fischlechner, M.; Courtois, F.; Lainé, C.; Hollfelder, F. Chem. Biol. 2012, 19, 10011009.

(10) Occhetta, P.; Centola, M.; Tonnarelli, B.; Redaelli, A.; Martin, I.; Rasponi, M. Sci. Rep. 2015, 5, 10288.

(11) Trantidou, T.; Friddin, M.; Elani, Y.; Brooks, N. J.; Law, R. V.; Seddon, J. M.; Ces, O. ACS Nano 2017, 11, 6549-6565.

(12) Elani, Y.; deMello, A. J.; Niu, X.; Ces, O. Lab Chip 2012, 12, 3514-3520.

(13) Fell, J. Eng. \& Technol. 2016, 11, 72-75.

(14) Ford, S. L. J. Int'l Com. \& Econ. 2014, 6, 40.

(15) Goyanes, A.; Wang, J.; Buanz, A.; Martínez-Pacheco, R.; Telford, R.; Gaisford, S.; Basit, A. W. Mol. Pharmaceutics 2015, 12, 4077-4084.

(16) Chen, R. K.; Jin, Y.-a.; Wensman, J.; Shih, A. Addit. Manuf. 2016, 12, 77-89.

(17) Dawood, A.; Marti, B. M.; Sauret-Jackson, V.; Darwood, A. Br Dent J 2015, 219, 521-529.

(18) Malik, H. H.; Darwood, A. R. J.; Shaunak, S.; Kulatilake, P.; El-Hilly, A. A.; Mulki, O.; Baskaradas, A. J. Surg. Res. 2015, 199, 512522.

(19) Nie, J.; Liang, Y.; Zhang, Y.; Le, S.; Li, D.; Zhang, S. Analyst 2013, 138, 671-676.

(20) Trantidou, T.; Regoutz, A.; Voon, X. N.; Payne, D. J.; Ces, O. Sens. Actuators, B 2018, 267, 34-41. 
(21) Kahl, L.; Molloy, J.; Patron, N.; Matthewman, C.; Haseloff, J.; Grewal, D.; Johnson, R.; Endy, D. Nat. Biotechnol. 2018, 36, 923.

(22) Kong, D. S.; Thorsen, T. A.; Babb, J.; Wick, S. T.; Gam, J. J.; Weiss, R.; Carr, P. A. Nat. Biotechnol. 2017, 35, 523.

(23) Kuss, M.; Duan, B. In Rapid Prototyping in Cardiac Disease: $3 D$ Printing the Heart, Farooqi, K. M., Ed.; Springer International Publishing: Cham, 2017, pp 167-182.

(24) Kundu, J.; Shim, J. H.; Jang, J.; Kim, S. W.; Cho, D. W. J. Tissue Eng. Regener. Med. 2015, 9, 1286-1297.

(25) Dimas, L. S.; Bratzel, G. H.; Eylon, I.; Buehler, M. J. Adv. Funct. Mater. 2013, 23, 4629-4638. 56.

(26) Heo, Y. J.; Takeuchi, S. Adv. Healthcare Mater. 2013, 2, 43-

(27) Elani, Y.; Law, R. V.; Ces, O. Nat. Commun. 2014, 5.

(28) Villar, G.; Graham, A. D.; Bayley, H. Science 2013, 340, 48 52.

(29) Funakoshi, K.; Suzuki, H.; Takeuchi, S. Anal. Chem. 2006, 78, 8169-8174.

(30) Bayley, H.; Cronin, B.; Heron, A.; Holden, M. A.; Hwang, W. L.; Syeda, R.; Thompson, J.; Wallace, M. Mol. BioSyst. 2008, 4, 1191 1208 .

(31) Karamdad, K.; Hindley, J.; Bolognesi, G.; Friddin, M. S.; Law, R. V.; Brooks, N. J.; Ces, O.; Elani, Y. Chem. Sci. 2018, 9, 4851-4858. (32) Elani, Y.; Gee, A.; Law, R. V.; Ces, O. Chem. Sci. 2013, 4, 3332-3338.

(33) Deng, N.-N.; Yelleswarapu, M.; Huck, W. T. J. Am. Chem. Soc. 2016, 138, 7584-7591.

(34) Hindley, J. W.; Elani, Y.; McGilvery, C. M.; Ali, S.; Bevan, C. L.; Law, R. V.; Ces, O. Nat. Commun. 2018, 9, 1093.

(35) Hadorn, M.; Boenzli, E.; Eggenberger Hotz, P.; Hanczyc, M. M. PLoS One 2012, 7, e50156. 808 .

(36) Villar, G.; Heron, A. J.; Bayley, H. Nat. Nano. 2011, 6, 803-

(37) Elani, Y.; Solvas, X. C. I.; Edel, J. B.; Law, R. V.; Ces, O. Chem. Commun. 2016, 52, 5961-5964.

(38) Baxani, D. K.; Morgan, A. J.; Jamieson, W. D.; Allender, C. J.; Barrow, D. A.; Castell, O. K. Angew. Chem. 2016, 128, 14452-14457.

(39) Trantidou, T.; Elani, Y.; Parsons, E.; Ces, O. Microsyst. Nanoeng. 2017, 3, 16091.

(40) Bolognesi, G.; Friddin, M. S.; Salehi-Reyhani, A.; Barlow, N. E.; Brooks, N. J.; Ces, O.; Elani, Y. Nat. Commun. 2018, 9, 1882.

(41) Thomas, J. M.; Friddin, M. S.; Ces, O.; Elani, Y. Chem. Commun. 2017, 53, 12282-12285.

(42) Armstrong, J. P. K.; Holme, M. N.; Stevens, M. M. ACS Nano 2017, 11, 69-83.

(43) Booth, M. J.; Schild, V. R.; Graham, A. D.; Olof, S. N.; Bayley, H. Sci. Adv. 2016, 2.

(44) Booth, M. J.; Restrepo Schild, V.; Box, S. J.; Bayley, H. Sci. Rep. 2017, 7, 9315.

(45) Tan, Y. C.; Hettiarachchi, K.; Siu, M.; Pan, Y. R.; Lee, A. P. J. Am. Chem. Soc. 2006, 128, 5656-5658.

(46) Elani, Y.; Trantidou, T.; Wylie, D.; Dekker, L.; Polizzi, K.; Law, R. V.; Ces, O. Sci. Rep. 2018, 8, 4564.

(47) Czekalska, M. A.; Kaminski, T. S.; Jakiela, S.; Sapra, K. T.; Bayley, H.; Garstecki, P. Lab Chip 2015, 15, 541-548.

(48) Tsuji, Y.; Kawano, R.; Osaki, T.; Kamiya, K.; Miki, N.; Takeuchi, S. Lab Chip 2013, 13, 1476-1481.

(49) Sarles, S. A.; Leo, D. J. Anal. Chem. 2010, 82, 959-966.

(50) Macdonald, N. P.; Cabot, J. M.; Smejkal, P.; Guijt, R. M.; Paull,

B.; Breadmore, M. C. Anal. Chem. 2017, 89, 3858-3866.

(51) Waheed, S.; Cabot, J. M.; Macdonald, N. P.; Lewis, T.; Guijt, R. M.; Paull, B.; Breadmore, M. C. Lab Chip 2016, 16, 1993-2013.

(52) Bhattacharjee, N.; Urrios, A.; Kang, S.; Folch, A. Lab Chip 2016, 16, 1720-1742.

(53) Au, A. K.; Huynh, W.; Horowitz, L. F.; Folch, A. Angew. Chem. Int. Ed. 2016, 55, 3862-3881.

(54) Mona, R.; Sumanpreet, C.; Dan, S.; Parameswaran, M. J. Micromech. Microeng. 2010, 20, 015026.

(55) Patko, D.; Mártonfalvi, Z.; Kovacs, B.; Vonderviszt, F.; Kellermayer, M.; Horvath, R. Sens. Actuators, B Chemical 2014, 196, 352-356.
(56) Trantidou, T.; Friddin, M. S.; Gan, K. B.; Han, L.; Bolognesi, G.; Brooks, N. J.; Ces, O. Anal. Chem. 2018, 90, 13915-13921.

(57) Pao, Y. H.; Rentzepis, P. M. Appl. Phy. Lett. 1965, 6, 93-95.

(58) Di Giacomo, R.; Krödel, S.; Maresca, B.; Benzoni, P.; Rusconi,

R.; Stocker, R.; Daraio, C. Sci. Rep. 2017, 7, 45897.

(59) Nelson, G.; Kirian, R. A.; Weierstall, U.; Zatsepin, N. A.; Faragó, T.; Baumbach, T.; Wilde, F.; Niesler, F. B.; Zimmer, B.; Ishigami, I. Opt. Express 2016, 24, 11515-11530.

(60) Hengsbach, S.; Lantada, A. D. Biomed. Microdevices 2014, 16, 617-627.

(61) King, P. H.; Jones, G.; Morgan, H.; de Planque, M. R.; Zauner, K.-P. Lab Chip 2014, 14, 722-729.

(62) Sarles, S. A.; Leo, D. J. Lab Chip 2010, 10, 710-717.

(63) Sarles, S. A.; Stiltner, L. J.; Williams, C. B.; Leo, D. J. ACS Appl. Mater. Interfaces 2010, 2, 3654-3663.

(64) El-Arabi, A. M.; Salazar, C. S.; Schmidt, J. J. Lab Chip 2012, $12,2409-2413$.

(65) Stachowiak, J. C.; Richmond, D. L.; Li, T. H.; Liu, A. P.; Parekh, S. H.; Fletcher, D. A. Proc Natl Acad Sci USA 2008, 105, $4697-$ 4702.

(66) Portonovo, S. A.; Schmidt, Biomed. Microdevices 2012, 14, 187-191.

(67) Barlow, N. E.; Smpokou, E.; Friddin, M. S.; Macey, R.; Gould, I. R.; Turnbull, C.; Flemming, A. J.; Brooks, N. J.; Ces, O.; Barter, L. M. C. Biomicrofluidics 2017, 11 .

(68) Barlow, N. E.; Bolognesi, G.; Flemming, A. J.; Brooks, N. J.; Barter, L. M. C.; Ces, O. Lab Chip 2016, 16, 4653-4657.

(69) Barlow, N. E.; Bolognesi, G.; Haylock, S.; Flemming, A. J.; Brooks, N. J.; Barter, L. M. C.; Ces, O. Sci. Reps. 2017, 7, 17551.

(70) Czekalska, M.; Kaminski, T.; Horka, M.; Jakiela, S.; Garstecki, P. Micromachines 2017, 8, 93.

(71) de Bruin, A.; Friddin, M. S.; Elani, Y.; Brooks, N. J.; Law, R.; Seddon, J. M.; Ces, O. RSC Adv. 2017, 7, 47796-47800.

(72) Chen, Z.; Han, J. Y.; Shumate, L.; Fedak, R.; DeVoe, D. L. Adv. Mater. Technol. 0, 1800511.

(73) Morgan, A. J. L.; Hidalgo San Jose, L.; Jamieson, W. D.; Wymant, J. M.; Song, B.; Stephens, P.; Barrow, D. A.; Castell, O. K. PLOS ONE 2016, 11, e0152023.

(74) Romanov, V.; Samuel, R.; Chaharlang, M.; Jafek, A. R.; Frost, A.; Gale, B. K. Anal. Chem. 2018, 90, 10450-10456.

(75) Gong, H.; Bickham, B. P.; Woolley, A. T.; Nordin, G. P. Lab Chip 2017, 17, 2899-2909.

(76) Olsen, M. H.; Hjortø, G. M.; Hansen, M.; Met, Ö.; Svane, I. M.; Larsen, N. B. Lab Chip 2013, 13, 4800-4809.

(77) Karamdad, K.; Law, R. V.; Seddon, J. M.; Brooks, N. J.; Ces, O. Lab Chip 2015, 15, 557-562.

(78) Gong, H.; Woolley, A. T.; Nordin, G. P. Lab Chip 2016, 16, 2450-2458.

(79) Lewis, J. A.; Ahn, B. Y. Nature 2015, 518, 42.

(80) Dryden, M. D. M.; Fobel, R.; Fobel, C.; Wheeler, A. R. Anal. Chem. 2017, 89, 4330-4338.

(81) Xia, Y.; Whitesides, G. M. Angew. Chem. 1998, 110, 568-594.

(82) Salehi-Reyhani, A.; Ces, O.; Elani, Y. Exp. Biol. Med. 2017, 242, 1309-1317. 
For TOC only

AFFORDABIIITY

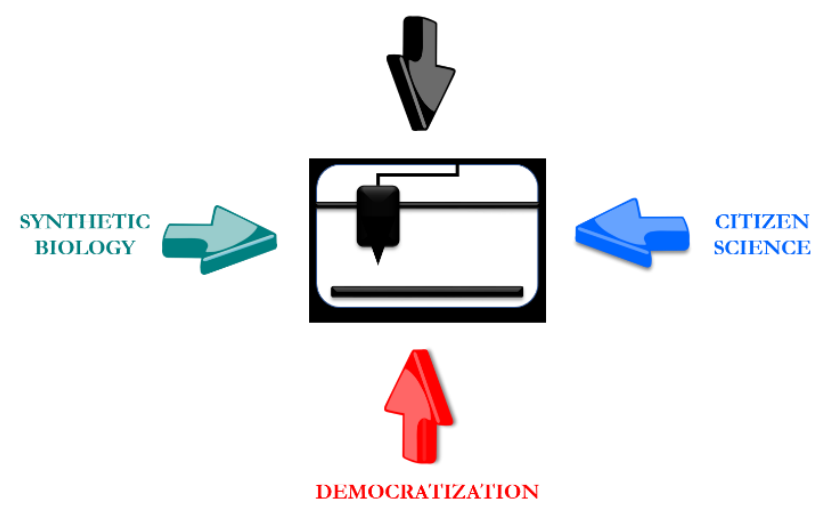

DEMOCRA'TIZA'IIION 